\title{
Assessment of different formation scenarios for the ring system of (10199) Chariklo (Corrigendum)
}

\author{
M. D. Melita ${ }^{1,2}$, R. Duffard ${ }^{3}$, J. L. Ortiz ${ }^{3}$, and A. Campo-Bagatin ${ }^{4,5}$ \\ ${ }^{1}$ Instituto de Astronomía y Física del Espacio (CONICET-UBA), CABA, 1428 Buenos Aires, Argentina \\ e-mail: melita@iafe.uba.ar \\ 2 Facultad de Ciencias Astronómicas y Geofísicas, Universidad Nacional de La Plata, 1900 FWA La Plata, Argentina \\ 3 Instituto de Astrofísica de Andalucía, IAA-CSIC, 18008 Granada, Spain \\ ${ }^{4}$ Departamento de Física, Ingeniería de Sistemas y Teoría de la Señal, Escuela Politécnica Superior, Universidad de Alicante, \\ 03690 Sant Vicent del Raspeig (Alacant), Spain \\ 5 Instituto de Física Aplicada a las Ciencias y la Tecnología, Universidad de Alicante, 03080 Alicante, Spain
}

A\&A 602, A27 (2017), DOI: 10.1051/0004-6361/201629858

Key words. Kuiper belt: general - minor planets, asteroids: general - minor planets, asteroid: individual: (10199) Chariklo errata, addenda

Some errors appeared in the published version.

The corrected Eq. (10) is:

$R_{a}=R_{b}\left(\sqrt{\left(\left(a_{\text {rings }} \Delta V_{b}^{2}\right) /\left(G M_{\mathrm{ch}}\right)\right)}-1\right)$.

In addition, the affiliation of J. L. Ortiz has been corrected, and the Acknowledgements section should read:

Acknowledgements. R.D. acknowledges the support of MINECO for his Ramón y Cajal Contract. Funding from Spanish funding form grant
AYA-2014-56637-C2-1-P is acknowledged, as is the Proyecto de Excelencia de la Junta de Andalucía, J. A. 2012-FQM1776. FEDER funds are also acknowledged. M.D.M. acknowledges the support of Min CyT ANPCyt PicT 1144/2013 and the support of the travel grant from CONICET and CSiC. The research leading to these results has received funding from the European Union's Horizon 2020 Research and Innovation Programme, under Grant Agreement No. 687378 